\title{
Oral Candidiasis Susceptibility of Mice Lacking Interferon Regulatory Factor 3, A Preliminary Report
}

\author{
Fahd Alsalleeh* \\ Department of Restorative Dental Sciences, Kingdom of Saudi Arabia \\ *Corresponding author: Fahd Alsalleeh, Restorative Dental Sciences Department, Saudi Arabia
}

\section{ARTICLE INFO

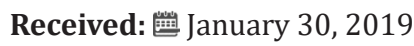 \\ Published: 幽 February 13, 2019}

Citation: Fahd Alsalleeh. Oral Candidiasis Susceptibility of Mice Lacking Interferon Regulatory Factor 3, A Preliminary Report. Biomed J Sci \& Tech Res 14(4)-2019. BJSTR. MS.ID.002571.

Keywords: C. albicans; Oral Candidiasis; IRF-3; Fungal infection; Type I Interferon

Abbreviations: PRRs: Pattern Recognition Receptors; IFNs: I Interferons; IRFS: IFN Regulatory Factors; YPD: Yeast Extract-Peptone-Dextrose; PBS: Phosphate Buffered Saline; CFU: Colony Forming Unit
ABSTRACT

The purpose of this research was to evaluate the significance of interferon regulatory factors 3 to the susceptibility of oral candidiasis in mice.

Materials and Methods: IRF- ${ }^{\mathrm{KO}}$ and C57BL/6 mice were injected with cortisone acetate subcutaneously in the dorsum of the neck and were used as the experimental groups. Mice with no cortisone acetate treatment were used as controls. Each mouse was inoculated by swabbing the oral cavity with a cotton swab soaked in PBS containing $10^{8}$ $\mathrm{CFU} / \mathrm{ml}$ of $C$. albicans. On days 1, 3, and six, a swab from the oral cavity of all mice was performed and plated on YPD agar to determine fungal burden. On day six post-infection, mice were sacrificed, and tongues were extracted and submitted for histology.

Results: The control mice, without cortisone acetate treatment had maintained their weight throughout the experiment. When both groups were treated with cortisone acetate, considerable body weight was lost. At day 3,5 , and 6, the IRF- $3^{\mathrm{K} 0}$ mice which were treated with cortisone acetate, a significant weight loss was observed compared to C57BL/6 mice. Mice that were treated with cortisone acetate had significant fungal burden at day 3 and 6. Microscopic analysis showed that fungal infection and hyphal formation invaded the superficial epithelial layer of the mucosa and the papillae in both experimental groups. The infiltration of neutrophils and eosinophils was observed more in IRF- $3^{\mathrm{KO}}$ mice which were treated with cortisone acetate compared C57BL/6 mice that received the same treatment. In conclusion, IRF-3 deficient mice are more susceptible to oral candidiasis if treated with cortisone acetate.

\section{Introduction}

Candidiasis is prevalent when the host defense is compromised. For example, individuals with diabetes mellitus, HIV infection, chronic systemic immune-suppressor drugs usage, or patients in intensive care units or having cancer are at high risk to develop persistent candida infection or recurrent episodes [1,2]. Oropharyngeal candidiasis, often known as the "thrush," is caused by ubiquitous commensal candida albicans (C. albicans) that irreversibly attaches and colonizes in the oral cavity. It is known that candidiasis is the most common oral lesion among HIV-infected individuals [3,4]. Moreover, a systematic review has indicated that in patients with cancer oral colonization with fungal organisms was found in $48.2 \%$ before treatment, $72.2 \%$ during treatment, and $70.1 \%$ after treatment. Also, $35 \%$ of Sjogren syndrome patients have oral candidiasis. In almost all cases, $C$. albicans was the dominant yeast. Therefore, there is an increased risk of clinically significant oral fungal infection. Current treatment focuses on prevention than understanding the mechanisms underlying the pathogenicity of the disease itself by prescribing antifungal drugs that often develop resistance [5].

Most of the studies have focused on using a mutant strain of mice with defects in Tymphocytes expressed CD+4 IL-17 that play a more central role in oral candidiasis, yet recognition of $C$. albicans and development of the adaptive immune response largely depends on cytokines produced by the innate immune cells. The mucosal surfaces and its cells are the first line of defense against many pathogens, including C. albicans. Mucosal epithelial, macrophages and dendritic cells recognize $C$. albicans through pattern recognition receptors (PRRs) and subsequently controlled by the production 
of cytokines [6]. Type I interferons (IFNs), a family of pleiotropic cytokines, play a significant role in the innate immune response at the mucosa.

Immunity to oral candidiasis relies upon the interactions between the innate and adaptive immune cells/cytokines. Several animal models have illustrated the role of type I IFN signaling to develop the appropriate immune response against extracellular pathogen and maintenance of mucosal immunity. Its induction is controlled by several downstream signaling in which ultimately result in the activation of the IFN regulatory factors (IRFs) [7]. IRF3 is considered a key transcription factor that ultimately induces expression of Type I IFN. Therefore, IRF-3 could thus be detrimental to the development of oral candidiasis. The purpose of this research was to evaluate the significance of IRF-3 to the susceptibility of oral candidiasis in mice.

\section{Material and Methods}

\section{Ethics Statement}

All animal experiments were conducted at the Animal Facility of the University of Nebraska Medical Center College of Dentistry and were approved by the Institutional Animal Care and Use Committee (IACUC protocol 13-024-04).

\section{C. albicans Preparation}

The C. albicans wild strain CA42 (courtesy of Dr. Audrey Atkin, University of Nebraska, Lincoln) was grown in yeast extractpeptone-dextrose (YPD) medium (Difco Laboratories, Detroit, Mich.) from fresh Sabouraud dextrose agar plates (Difco) and incubated for $24 \mathrm{~h}$ at $37{ }^{\circ} \mathrm{C}$ in a shaker at 250rpm. Cells were harvested and washed twice with phosphate buffered saline (PBS). Cells were then re-suspended in $10 \mathrm{~mL}$ of PBS, counted following serial dilution, standardized, and diluted to a final concentration of $10^{8} \mathrm{CFU} / \mathrm{ml}$.

\section{Mouse Oral Candidiasis Model}

IRF-3KO mice on the B6 background were offspring of breeder pairs obtained from Prof. Tom Petro University of Nebraska Medical Center. The C57BL/6 mice were purchased from Harlan Sprague Dawley and used at 8-10 weeks of age. All mice were screened for the presence of $C$. albicans before experimenting on day 0 by swabbing the oral cavity with a sterile cotton swab and plating on YPD agar. To determine the role of IRF-3 on the development of oral candidiasis, mice were divided into the following groups $(n=10)$ :

a) C57BL/6 mice immunocompetent were infected with $C$. albicans as a negative control.

b) C57BL/6 mice immunocompetent were injected with optimized dose $(200 \mathrm{mg} / \mathrm{kg})$ in a total volume of $0.2 \mathrm{~mL}$ of cortisone acetate (Sigma-Aldrich) in PBS and $0.1 \%$ tween 80 subcutaneously in the dorsum of the neck before, one day and three days after infection Then mice were treated as in group $\mathrm{A}$.

c) $\mathrm{IRF}-3^{\mathrm{KO}}$ were treated as group $\mathrm{A}$.

d) IRF- $3^{\mathrm{KO}}$ were treated as group B.

Each mouse was inoculated by swabbing all surfaces of the oral cavity with a cotton swab soaked in PBS containing $108 \mathrm{CFU} / \mathrm{ml}$ of C. albicans. Mice were caged individually after infection, and their weight was recorded daily.

\section{Determination of Fungal Burden}

On days 1, 3, and 6 a swab from the oral cavity of all mice with sterile cotton and plated on YPD agar to determine fungal burden. Each swab was plated across the surface of a $100 \mathrm{~cm}^{2}$ YPD agar plate. Each plate was incubated aerobically at $37^{\circ} \mathrm{C}$ for $48 \mathrm{hr}$, and CFUs were determined by counting.

\section{Histology}

On day six post-infection, mice were sacrificed, and tongues were extracted and submitted for histology. Extracted tongues were submerged in $10 \%$ formalin and left at room temperature and protected from light for $72 \mathrm{hr}$ and embedded in paraffin. Tongues were cut into sagittal sections and stained with Hematoxylin and Eosin or Periodic Acid Schiff. Tongues were imaged under light microscopy at 40X magnification.

\section{Results}

The immunocompetent and IRF- $3^{\mathrm{KO}}$ mice; group $\mathrm{A}$ and $\mathrm{C}$ respectively, without cortisone acetate treatment had maintained their weight throughout the experiment. When both groups were treated with cortisone acetate; group B and D respectively, considerable body weight was lost and was significant from other groups (Figure 1). At day 3,5, and 6, the IRF- $3^{\mathrm{KO}}$ mice which were treated with cortisone acetate; group D, a significant weight loss was observed compared to immunocompetent mice which were treated with cortisone acetate; group B. Mice that were treated with cortisone acetate; group B and D had significant fungal burden at day 3 and 6 . IRF- $3^{\text {ко }}$ mice which were treated with cortisone acetate; group D, had $3.2 \times 10^{3} \mathrm{CFU} / \mathrm{g}$ compared to $2.2 \times 10^{2} \mathrm{CFU} / \mathrm{g}$ in immunocompetent mice which treated with cortisone acetate, group B (Figure 2). Microscopic analysis (Periodic Acid Schiff) showed that fungal infection and hyphal formation invaded the superficial epithelial layer of the mucosa and the papillae in both group B and D (Figure 3). The infiltration of neutrophils and eosinophils was observed more in IRF- $3^{\mathrm{KO}}$ mice which were treated with cortisone acetate compared to immunocompetent mice in group B. 


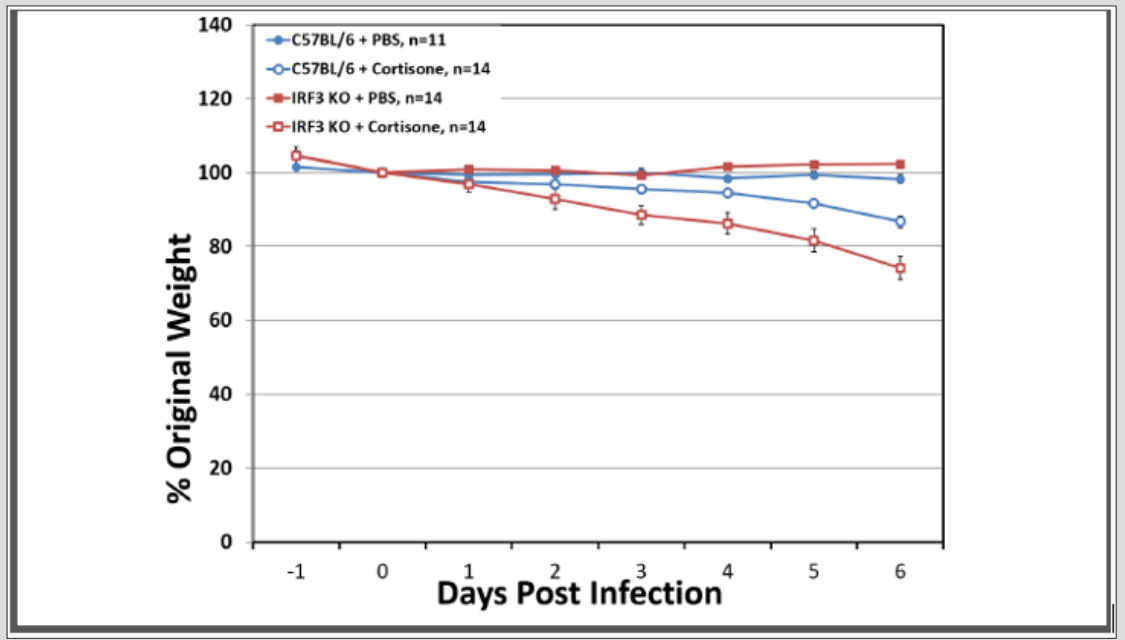

Figure 1: IRF-3 ${ }^{\mathrm{KO}}$ and C57BL/6 mice followed by oral infection with C. albicans with and without cortisone treatment. Weight was assessed daily and is shown as mean $\%$ of starting weight.

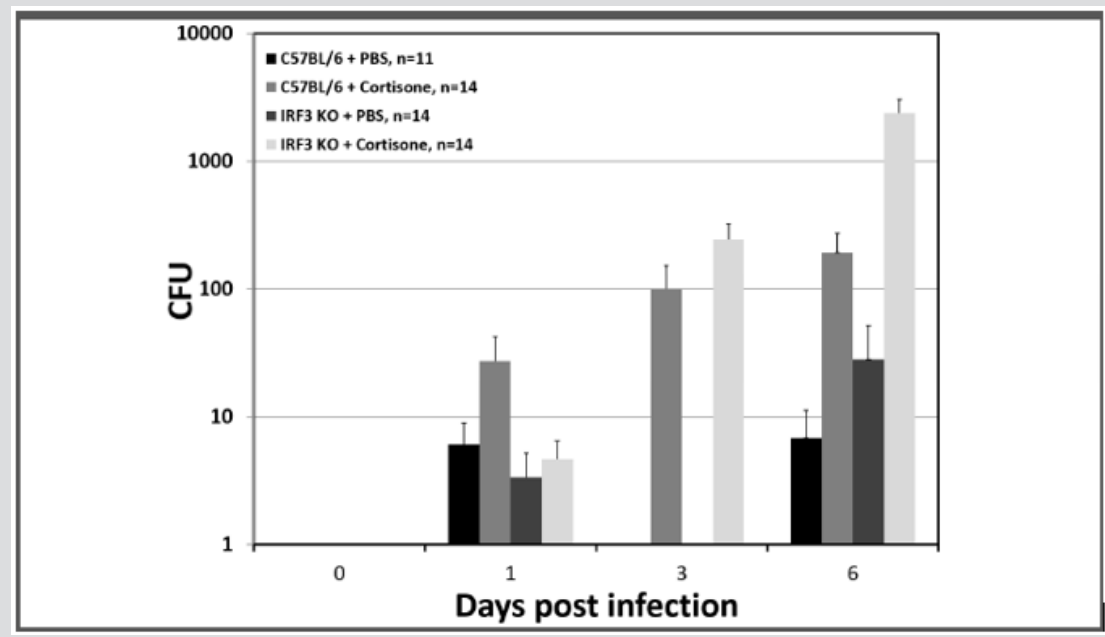

Figure 2: IRF-3 ${ }^{\mathrm{KO}}$ and C57BL/ 6 mice followed by oral infection with C. albicans with and without cortisone treatment. On days 1,3 , and 6 , fungal loads were assessed by CFU of the oral cavity. Bars show the geometric mean.

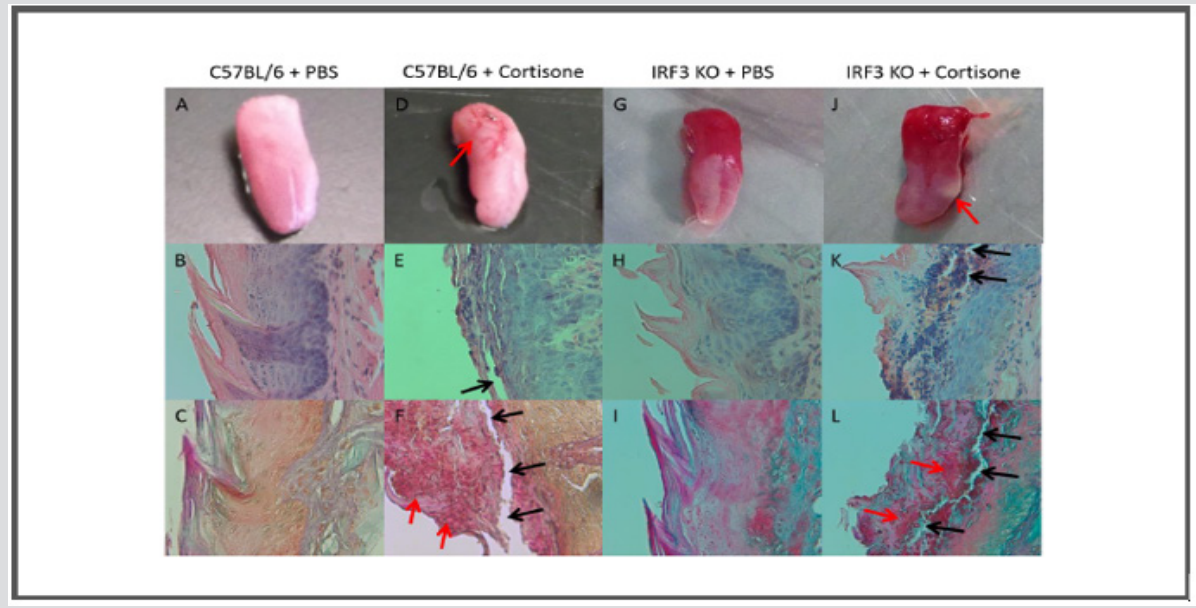

Figure 3: Tongues $(A, D, G, J)$ of a representative mouse from each group were cut into sagittal sections and stained with Hematoxylin (B, E, H, K) and Eosin or Periodic Acid Schiff (C, F, I, L). Tongues were imaged under light microscopy at 40X magnification. 


\section{Discussion}

C. albicans is considered a benign microorganism and present in $50 \%$ of healthy asymptomatic individuals [8]. However, oropharyngeal candidiasis "thrush," is caused by ubiquitous commensal C. albicans that irreversibly attaches and colonizes in the oral cavity. Several autoimmune diseases and drugs were cited to be predisposing factors that facilitate the development and evasion of C. albicans infection of mucosal surfaces $[3,5]$. The innate immune response against $C$. albicans is detrimental that eventually tolerate or invade the growth of infection to cause the disease $[9,10]$. The response is rapid and controlled by transcription factors, followed by production of cytokines [6]. Among these cytokines, type I interferons (IFNs), IRF-3 is considered a key transcription factor that ultimately induces expression of Type I IFN. Therefore, the present study aimed to investigate the involvement of IRF-in the development of oral candidiasis in vivo.

The result presented for the first time that IRF-3 in deficiency worsens the disease process in mice. The lack of IRF-3 itself was not enough to establish the disease. The role of IFNs in host protection against $C$. albicans is well established and have reviewed recently [11]. T lymphocytes expressed CD+4 IL-17 play a more central role in oral candidiasis. The induction of this subset of $\mathrm{T}$ lymphocytes will subsequently induce the production of IFNs. It is reasonable to conclude that mice lack IRF-3; a key transcription factor of IFN expression had affected the function of CD+4 IL-17 lymphocytes. The results herein are consistent with previous reports that showed IFNs enhanced C. albicans clearance [12]. In conclusion, IRF-3 deficient mice are more susceptible to oral candidiasis. Further studies are needed to analyze the down/up-stream signaling pathways during the disease process. In the future, adjuvant therapy for treatment of oral candidiasis may target manipulation of cytokine gene expression.

\section{ISSN: 2574-1241}

DOI: 10.26717.BJSTR.2019.14.002571

Fahd Alsalleeh.Biomed J Sci \& Tech Res

This work is licensed under Creative Commons Attribution 4.0 License

Submission Link: https://biomedres.us/submit-manuscript.php

\section{Acknowledgment}

This work was funded by seeding grant of University of Nebraska Medical Center.

\section{References}

1. Falagas ME, Apostolou KE, Pappas VD (2006) Attributable mortality of candidemia: a systematic review of matched cohort and case-control studies. Eur J Clin Microbiol Infect Dis 25(7): 419-425.

2. Conti HR, Gaffen SL (2010) Host responses to Candida albicans: Th17 cells and mucosal candidiasis. Microbes Infect 12(7): 518-527.

3. Rex JH, Walsh TJ, Sobel JD, Filler SG, Pappas PG, et al. (2000) Practice guidelines for the treatment of candidiasis. Infectious Diseases Society of America. Clin Infect Dis 30(4): 662-678.

4. Ramos ESM, Lima CM, Schechtman RC, Trope BM, Carneiro S (2010) Superficial mycoses in immunodepressed patients (AIDS). Clin Dermatol 28(2): 217-225.

5. Pappas PG, Rex JH, Sobel JD, Filler SG, Dismukes WE, et al. (2004) Guidelines for treatment of candidiasis. Clin Infect Dis 38(2): 161-189.

6. Filler SG (2006) Candida-host cell receptor-ligand interactions. Curr Opin Microbiol 9(4): 333-339.

7. Chen K, Liu J, Cao X (2017) Regulation of type I interferon signaling in immunity and inflammation: A comprehensive review. Journal of autoimmunity 83: 1-11.

8. Hebecker B, Naglik JR, Hube B, Jacobsen ID (2014) Pathogenicity mechanisms and host response during oral Candida albicans infections. Expert review of anti-infective therapy 12(7): 867-879.

9. Weindl G, Wagener J, Schaller M (2011) Interaction of the mucosal barrier with accessory immune cells during fungal infection. International journal of medical microbiology: IJMM 301(5): 431-435.

10. Cheng SC, Joosten LA, Kullberg BJ, Netea MG (2012) Interplay between Candida albicans and the mammalian innate host defense. Infect Immun 80(4): 1304-1313.

11. Gozalbo D, Maneu V, Gil ML (2014) Role of IFN-gamma in immune responses to Candida albicans infections. Front Biosci (Landmark Ed) 19: $1279-1290$

12. Mencacci A, Spaccapelo R, Del Sero G, Enssle KH, Cassone A, et al. (1996) CD4+ T-helper-cell responses in mice with low-level Candida albicans infection. Infect Immun 64(12): 4907-4914.

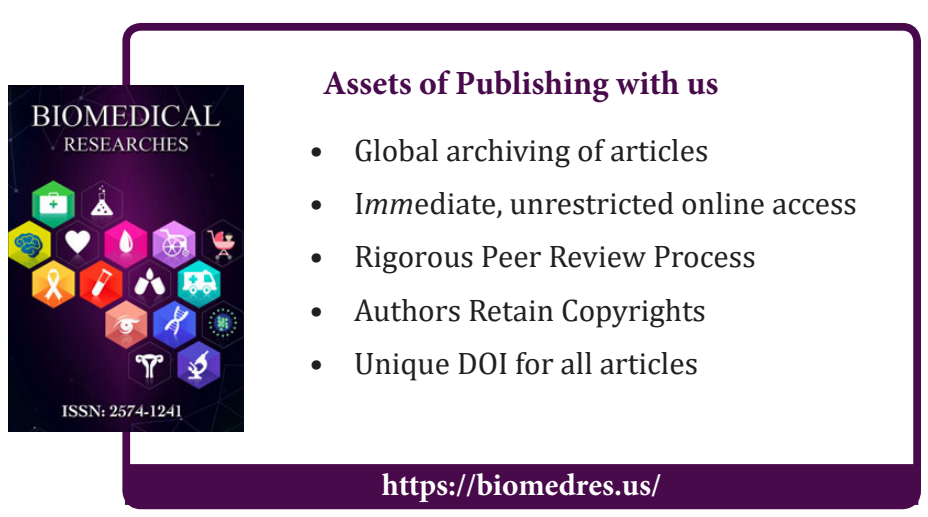

